\title{
Note
}

\section{Coffee Diterpenes Potentiate the Cytolytic Activity of KHYG-1 NK Leukemia Cells}

\author{
Takeshi Saito, Daigo Abe and Yoichi Nogata ${ }^{*}$ \\ NARO Western Region Agricultural Research Center, 1-3-1, Senyu-cho, Zentsuji, Kagawa 765-8508, Japan
}

Received November 28, 2014 ; Accepted January 4, 2015

\begin{abstract}
Natural killer (NK) cells play a critical role in early host defense against tumor and virus-infected cells. Here, we report that two coffee diterpenes, cafestol and kahweol, potentiate the cytotoxic activity of KHYG-1, an NK leukemia cell line, against the NK-sensitive cell line K562. Western blot analysis and quantitative real-time PCR indicated that cafestol and kahweol markedly enhanced the expression of granzyme B. While both diterpenes had no or little effect on the expression of other cytotoxic effector molecules, kahweol elevated the phosphorylated forms of ATF-2, c-Jun and CREB transcription factors, which might participate in granzyme $B$ gene expression. These results suggested that cafestol and kahweol upregulate granzyme B expression by activating transcription factors, leading to enhanced cytolytic activity of NK cells.
\end{abstract}

Keywords: cafestol, granzyme B, kahweol, natural killer cell

\section{Introduction}

Cafestol and kahweol are structurally related diterpene molecules contained in coffee beans. Cafestol is found in both arabica and robusta beans, while kahweol is only found in arabica beans. The concentrations of these diterpenes in coffee beverages are $6-12 \mathrm{mg} /$ cup of unfiltered coffee and $0.2-0.6 \mathrm{mg} / \mathrm{cup}$ of filtered coffee (Urgert et al., 1995; Gross et al., 1997). There are several reports detailing the adverse effects of coffee diterpenes; for example, cafestol and kahweol raise serum cholesterol (Weusten-Van der Wouw et al., 1994; Urgert et al., 1996) or serum ALT and AST levels (Urgert et al., 1997; Boekschoten et al., 2004). In contrast, beneficial effects of coffee diterpenes have also been reported. For example, kahweol prevents osteoclastogenesis (Fumimoto et al., 2012), and cafestol and kahweol exhibit anticarcinogenic properties (Cavin et al., 2002).

Natural killer (NK) cells are the first line of defense against tumor and virus-infected cells without prior antigenic stimulation. NK cells have cytolytic granules that contain cytotoxic effector molecules. The serine protease granzyme B, a cytotoxic effector, plays a primary role in NK-mediated cytotoxicity. Upon arrival at target cells, NK cells release the cytotoxic effectors and induce the target cells to undergo apoptosis.

We have explored compounds from natural sources that potentiate NK activity using the NK leukemia cell line KHYG-1 (Yagita et al., 2000). Here, we found that cafestol and kahweol enhance the cytolytic activity of NK cells, likely through the upregulation of granzyme B.

\section{Materials and Methods}

Materials Cafestol and kahweol (Fig. 1A) were purchased from Funakoshi (Tokyo, Japan). The NK leukemia cell line KHYG-1 and NK-sensitive cell line K562 were purchased from the Japanese Collection of Research Bioresources Cell Bank (Osaka, Japan). Recombinant human IL-2 was purchased from Wako Pure Chemical Industries, Ltd. (Osaka, Japan). Fetal bovine serum (FBS) was from Biological Industries (Beit-Haemek, Israel). FastStart Universal SYBR Green Master (ROX) was from Roche Applied Science (Indianapolis, IN, USA). Antibodies against $\beta$-actin $(1: 3,000)$, granzyme A $(1: 3,000)$, granzyme B $(1: 3,000)$, Fas ligand (1:1,000), ATF-2 (1:1,000), phospho-ATF-2 (Thr69/71, 1:500), c-Jun (1:1,000), phospho-c-Jun (Ser73, 1:3,000), CREB $(1: 1,000)$, phospho-CREB (Ser133, 1:2,000) and HRP-linked antirabbit $\operatorname{IgG}(1: 3,000)$ were obtained from Cell Signaling Technology 
(Beverly, MA, USA). Antibody against Perforin was from Abcam Inc. (Cambridge, MA, USA).

Cell culture KHYG-1 cells were cultured in RPMI-1640 supplemented with $10 \%$ FBS, $50 \mathrm{ng} / \mathrm{mL}$ human IL-2, 100 units $/ \mathrm{mL}$ penicillin $\mathrm{G}$, and $100 \mu \mathrm{g} / \mathrm{mL}$ streptomycin sulfate. K562 cells were cultured in RPMI-1640 supplemented with $10 \%$ FBS, 100 units $/ \mathrm{mL}$ penicillin $\mathrm{G}$, and $100 \mu \mathrm{g} / \mathrm{mL}$ streptomycin sulfate.

Cytotoxicity assays KHYG-1 cells were cultured in the presence or absence of each compound for the indicated times, and then were collected by centrifugation and resuspended in RPMI-1640 supplemented with 1\% FBS. K562 cells were also collected and resuspended in RPMI-1640 supplemented with 1\% FBS. Then, KHYG-1 and K562 cells were co-cultured for $4 \mathrm{~h}$ at the indicated effector-target ratios. NK cell-mediated cytotoxicity was determined by measuring lactate dehydrogenase (LDH) release from K562 cells using a Cytotoxicity Detection Kit (Roche Applied Science) according to the manufacturer's instructions.

Quantitative real-time PCR Total RNA was purified using a High Pure RNA Isolation Kit (Roche Applied Science). Purified RNAs were reverse transcribed using a Primescript RT reagent kit (Takara Bio, Shiga, Japan) to obtain cDNA. SYBR Green-based quantitative real-time PCR was performed using an ABI 7500 Real-Time PCR System (Life Technologies, Gaithersburg, MD, USA) according to the manufacturer's protocol. Primer sequences used in the real-time PCR were as follows:

GAPDH, 5' - CA A GGCTGTGGGCAAGGT-3' and 5' - G G A A G GCCA T GCCAGT GA-3'; perforin, $5^{\prime}$ - T G T C G A G G C C C A G G T C A A - 3' a n d 5' - CCTTGGCTTCGGCAGAGAT-3'; granzyme a, 5' - C G T G C A A T G G A G A T T C T G GA A - 3' and 5'-GGAAGTGA C C C C TCGGAAA - 3'; granzymeb, 5' - G - C C C C T A C A T GGC T TA TCT TA TGA - 3' and 5' - GAAGCCACCGCACCTCT TC-3'; fas ligand, 5 ' - C C T T G A C A C C T C A G C C T C T A C A - 3' a n d 5'-CAGGCCAGCCCCAGCAAAC-3'. Data analyses were performed with 7500 System SDS software version 1.3.1 (Life Technologies).

Immunoblotting Proteins were extracted from KHYG-1 cells using M-PER Mammalian Protein Extraction Reagent (Thermo Scientific, Rockford, IL, USA) in the presence of phosphatase inhibitor cocktail and protease inhibitor cocktail (Wako Pure Chemical Industries, Ltd). Equal amounts of protein were resolved by SDS-PAGE and transferred to Immobilon-P PVDF membranes (Merck Millipore, Billerica, MA, USA). The blots were probed with the indicated primary antibodies for $1 \mathrm{~h}$, and then washed with TBS-T and incubated with HRP-linked secondary antibodies for $1 \mathrm{~h}$ at room temperature. The immunoreactive protein was visualized using an ECL-Select Western Blotting Detection System (GE Healthcare Japan, Tokyo, Japan).

Cytokine measurement The levels of IFN- $\gamma$ in the culture supernatant of KHYG-1 cells were determined using a LEGEND MAX Human IFN- $\gamma$ ELISA Kit (BioLegend, San Diego, CA,
USA) according to the manufacturer's protocol.

Statistical analysis Results were expressed as the mean \pm SD. Statistical analyses of these data were conducted using a Student's $t$-test. A value of $P<0.05$ was considered statistically significant.

\section{Results and Discussion}

To explore the compounds that potentiate NK cell function, KHYG-1 cells were cultured in the presence of a variety of natural products for $72 \mathrm{~h}$. Evaluation of cytolytic activity was determined by LDH activity derived from K562 target cells. As shown in Fig. $1 \mathrm{~B}$, cafestol and kahweol significantly potentiated the cytolytic activity of KHYG-1 cells. At an effector:target (E:T) ratio of 10:1, cafestol and kahweol $(30 \mu \mathrm{M})$ enhanced the cytolytic activity by 1.4- or 1.5-fold, respectively. In comparison with cafestol, kahweol exhibited a strong effect on KHYG-1 cytolytic activity.

Because NK cells are known to produce IFN- $\gamma$, which contributes to antiviral defense, we tested whether coffee diterpenes enhance the production of IFN- $\gamma$. KHYG-1 cells were cultured for $24 \mathrm{~h}$ in the presence of $30 \mu \mathrm{M}$ of cafestol or kahweol, and then IFN- $\gamma$
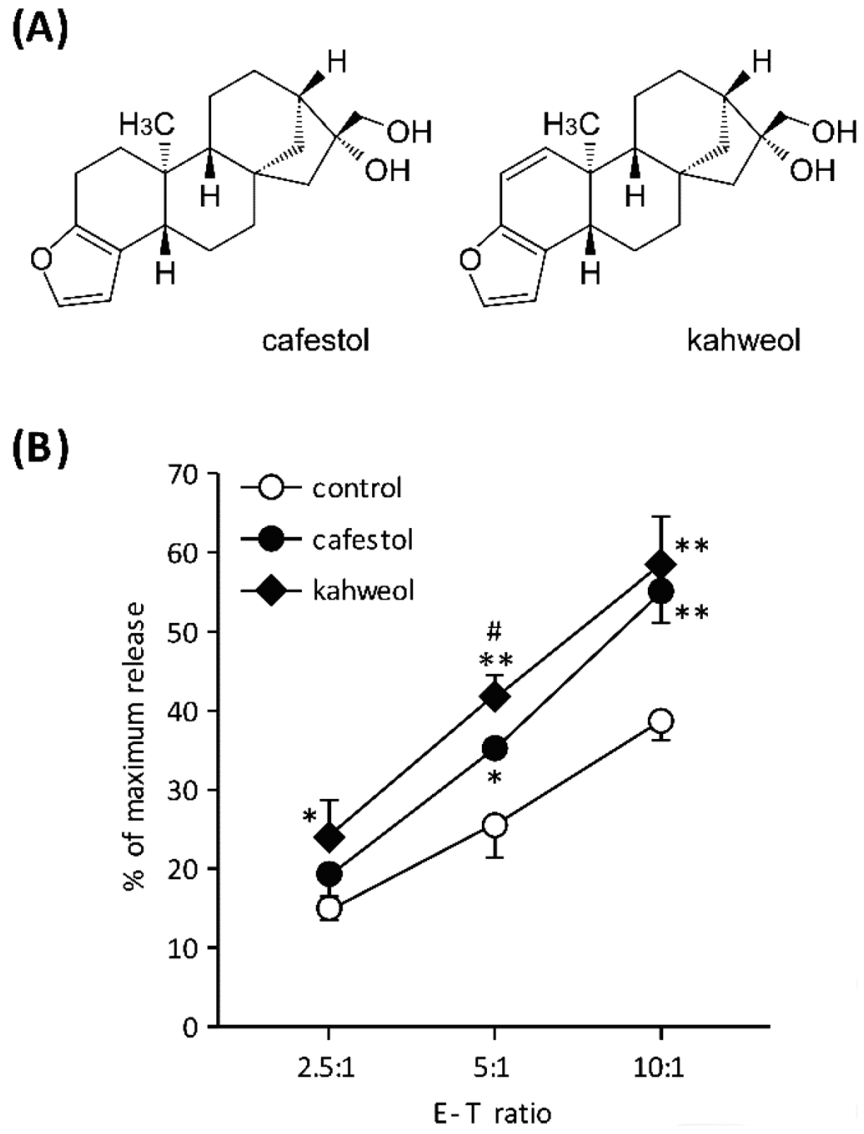

Fig. 1. Cytolytic activity of KHYG-1 cells. (A) Structure of cafestol and kahweol. (B) Lysis of K562 target cells by KHYG-1 effector cells. KHYG-1 cells were cultured for $72 \mathrm{~h}$ in the presence of cafestol or kahweol (both at $30 \mu \mathrm{M}$ ). KHYG-1 cells and K562 cells were washed and co-cultured for $4 \mathrm{~h}$ at the indicated effector:target ratios. Cytotoxicity was determined by LDH release from K562 cells. DMSO was used as a vehicle control. Values represent the mean $\pm \mathrm{SD}(\mathrm{n}=3)$. ${ }^{*} P<0.05,{ }^{*} P<0.01$, compared with vehicle control. ${ }^{*} P<0.05$, compared with cafestol. 
levels in the supernatants were measured by ELISA. The results showed cafestol and kahweol did not enhance the production of IFN- $\gamma$ (Fig. 2A). Next, to examine the function of cafestol and kahweol on potentiated cytolytic activity of KHYG-1 cells, the levels of proteins responsible for the cytolytic activity of NK cells were analyzed. KHYG-1 cells were treated with cafestol or kahweol for $72 \mathrm{~h}$, and then the protein levels of cytotoxic effectors were examined by western blot analysis. As shown in Fig. 2B, cafestol and kahweol moderately enhanced the protein levels of Fas ligand and granzyme A. However, both had little effect on the level of perforin, a cytotoxic effector that forms pore-like structures on the target cells. It is noteworthy that cafestol and kahweol markedly enhanced the protein levels of granzyme B at a dose of $30 \mu \mathrm{M}$. We also examined whether the gene expression of cytotoxic effectors are induced by cafestol and kahweol. Quantitative real-time PCR results showed that cafestol and kahweol also markedly enhanced the mRNA levels of granzyme B (Fig. 2C). Similar to the cytolytic activity results, kahweol induced a significantly higher level of granzyme B expression than cafestol. The expression of perforin, Fas ligand and granzyme A by cafestol and kahweol were significant, but underwent smaller increases (Fig. 2C). The mechanism underlying the upregulation of Fas ligand and granzyme A induced by cafestol and kahweol will need to be addressed in future studies. These results suggest that cafestol and kahweol potentiated NK cytolytic activity by enhancing the expression of cytotoxic effectors. In particular, marked increases in granzyme B by cafestol and kahweol seemed to be critical for the increase in KHYG-1 cytolytic activity.

It is known that upstream of the human and mouse granzyme B transcription start site contains functional binding sites for transcription factors, such as CREB (cAMP responsive elementbinding protein) and AP-1 (Hanson et al., 1993; Babichuk et al., 1997). Therefore, we examined whether the activation of transcription factors that likely participate in the expression of granzyme B is regulated by kahweol. The results of a time-course analysis indicated that kahweol-induced increases of granzyme B were observed at $24 \mathrm{~h}$. Of note, kahweol increased the phosphorylated/activated form of ATF-2 and c-Jun earlier than $24 \mathrm{~h}$ (Fig. 3). Additionally, kahweol increased the phosphorylated form and total protein content of CREB at $0.5 \mathrm{~h}$ (Fig. 3). ATF-2 forms homo- and heterodimers with c-Jun or CREB and binds to CRE (Hai et al., 1991). Furthermore, c-Jun can form homodimers and binds to CRE and AP-1, both of which regulate granzyme B expression (Hai et al., 1991). These results suggested that the increase in the activated-forms of the transcription factors induced by kahweol contribute to the upregulation of granzyme B gene. It is known that both ATF-2 and c-Jun are phosphorylated by the
(A)

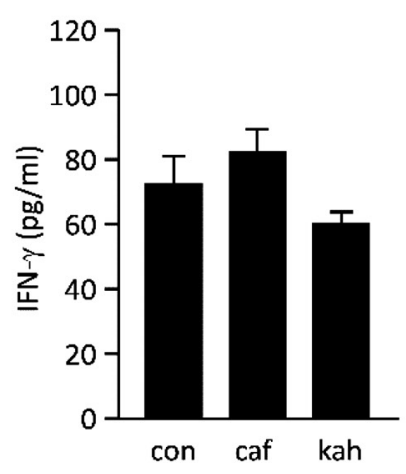

(C)

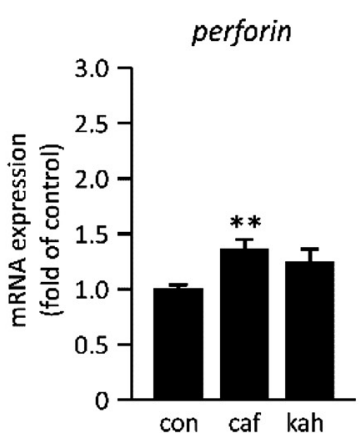

(B)

\section{, \\ Perforin \\ Fas ligand \\ Granzyme A \\ Granzyme B \\ $\beta$-Actin}
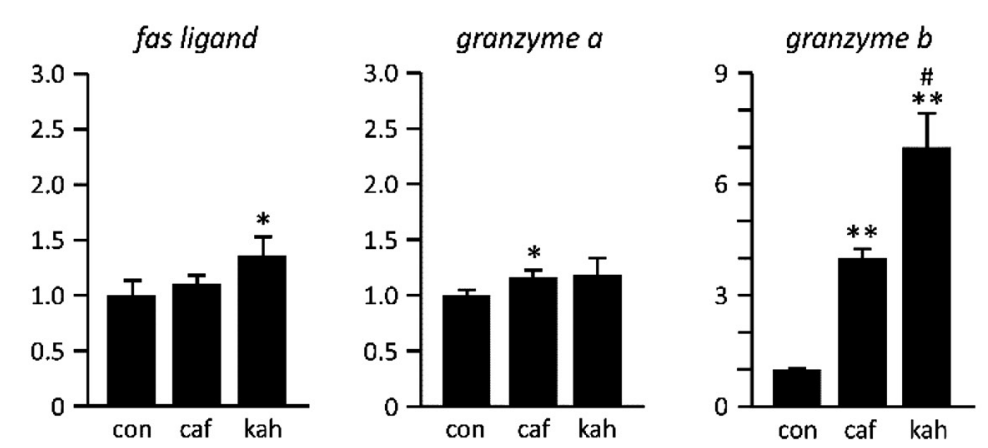

Fig. 2. Expression of IFN $\gamma$ and cytotoxic effectors. (A) Levels of IFN $\gamma$ in the culture supernatant of KHYG-1 cells treated with $30 \mu \mathrm{M}$ of cafestol (caf) or kahweol (kah) for $24 \mathrm{~h}$ were determined by ELISA. DMSO was used as a vehicle control (con). (B) Western blot analysis for the cytotoxic effectors. KHYG-1 cells were treated with the indicated doses of cafestol and kahweol for $72 \mathrm{~h}$. Duplicate lanes in immunoblots are derived from individual culture wells. (C) The mRNA levels of cytotoxic effectors in KHYG-1 cells treated with $30 \mu \mathrm{M}$ of cafestol or kahweol for $72 \mathrm{~h}$ were quantified by real-time PCR. Values represent the mean $\pm \mathrm{SD}(\mathrm{n}=3) . * P<0.05,{ }^{*} P<0.01$, compared with vehicle control (con). ${ }^{*} P<0.05$, compared with cafestol. 


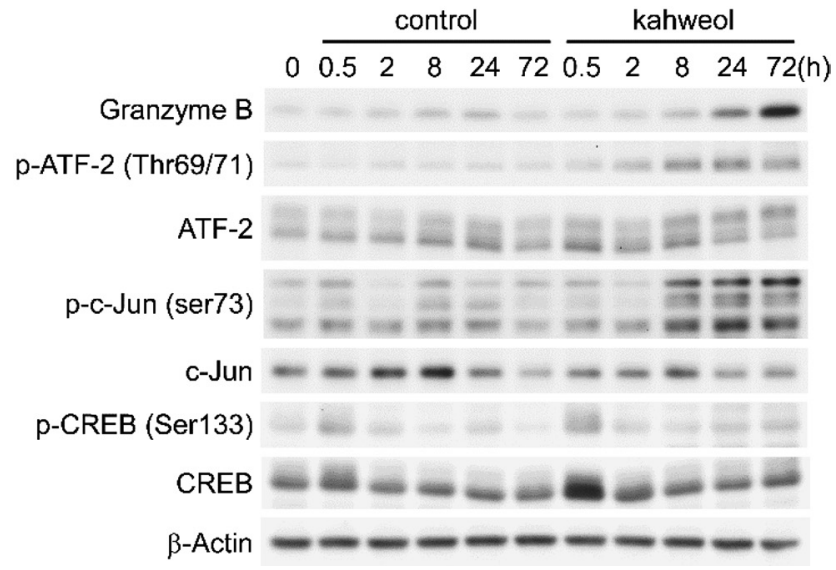

Fig. 3. Effect of kahweol on the activation of transcription factors. KHYG-1 cells were treated with $30 \mu \mathrm{M}$ of kahweol for the indicated times. DMSO was used as a vehicle control. Levels of total and phosphorylated ( $\mathrm{p}$ ) proteins were determined by western blot analysis.

upstream kinase SAPK/JNK. Additionally, ATF-2 is also known to be phosphorylated by p38 MAPK (Davis, 2000). Therefore, it is possible that cafestol and kahweol activate these kinases, leading to activation of ATF-2 and c-Jun. However, it remains unclear whether these transcription factors actually participate in the expression of granzyme B in KHYG-1 cells. Therefore, detailed characterization of the upregulation of granzyme B brought about by cafestol and kahweol should be addressed.

In conclusion, the coffee diterpenes kahweol and cafestol increase the level of granzyme B by activating the transcription factors that regulate the granzyme $\mathrm{B}$ gene promoter. The increased levels of granzyme B might contribute to the enhanced cytolytic activity of KHYG-1 cells. These effects of coffee diterpenes are expected to be beneficial for maintaining animal and human health.

\section{References}

Babichuk C.K. and Bleackley R.C. (1997). Mutational analysis of the murine granzyme B gene promoter in primary $\mathrm{T}$ cells and a $\mathrm{T}$ cell clone. J. Biol. Chem., 272, 18564-18571.

Boekschoten M.V., Schouten E.G., and Katan M.B. (2004). Coffee bean extracts rich and poor in kahweol both give rise to elevation of liver enzymes in healthy volunteers. Nutr. J., 3, 7.

Cavin C., Holzhaeuser D., Scharf G., Constable A., Huber W.W., and Schilter B. (2002). Cafestol and kahweol, two coffee specific diterpenes with anticarcinogenic activity. Food Chem. Toxicol., 40, 1155-1163.

Davis R.J. (2000). Signal transduction by the JNK group of MAP kinases. Cell, 103, 239-252.

Fumimoto R., Sakai E., Yamaguchi Y., Sakamoto H., Fukuma Y., Nishishita K., Okamoto K., and Tsukuba T. (2012). The coffee diterpene kahweol prevents osteoclastogenesis via impairment of NFATc1 expression and blocking of Erk phosphorylation. J. Pharmacol. Sci., 118, $479-486$.

Gross G., Jaccaud E., and Huggett A.C. (1997). Analysis of the content of the diterpenes cafestol and kahweol in coffee brews. Food Chem. Toxicol., 35, 547-554.

Hai T. and Curran T. (1991). Cross-family dimerization of transcription factors Fos/Jun and ATF/CREB alters DNA binding specificity. Proc. Natl. Acad. Sci. U.S.A., 88, 3720-3724.

Hanson R.D., Grisolano J.L., and Ley T.J. (1993). Consensus AP-1 and CRE motifs upstream from the human cytotoxic serine protease B (CSP-B/ CGL-1) gene synergize to activate transcription. Blood, 82, 2749-2757.

Urgert R., van der Weg G., Kosmeijer-Schuil T.G., van de Bovenkamp P., Hovenier R., and Katan M.B. (1995). Levels of the cholesterol-elevating diterpenes cafestol and kahweol in various coffee brews. J. Agric. Food Chem., 43, 2167-2172.

Urgert R. and Katan M.B. (1996). The cholesterol-raising factor from coffee beans. J. R. Soc. Med., 89, 618-623.

Urgert R., Essed N., van der Weg G., Kosmeijer-Schuil T.G., and Katan M.B. (1997). Separate effects of the coffee diterpenes cafestol and kahweol on serum lipids and liver aminotransferases. Am. J. Clin. Nutr., 65, 519-524.

Weusten-Van der Wouw M.P., Katan M.B., Viani R., Huggett A.C., Liardon R., Liardon R., Lund-Larsen P.G., Thelle D.S., Ahola I., Aro A., Meyboom S., and Beynen A.C. (2004). Identity of the cholesterol-raising factor from boiled coffee and its effects on liver function enzymes. $J$. Lipid Res., 35, 721-733.

Yagita M., Huang C.L., Umehara H., Matsuo Y., Tabata R., Miyake M., Konaka Y., and Takatsuki K. (2000). A novel natural killer cell line (KHYG-1) from a patient with aggressive natural killer cell leukemia carrying a p53 point mutation. Leukemia, 14, 922-930. 